\title{
Voltage plateaus on $V(I)$ curves of long quasi-one-dimensional superconducting wires (without microwave irradiation)
}

\author{
V.I. Kuznetsov* and A. A. Firsov \\ Institute of Microelectronics Technology and High Purity Materials, \\ Russian Academy of Sciences, Chernogolovka, Moscow Region 142432, Russia
}

(Dated: August 13, 2019)

\begin{abstract}
Almost constant voltage plateaus on the $V(I)$ curves of long quasi-one-dimensional superconducting aluminum wires placed in magnetic field at temperatures $T$ slightly below the critical superconducting temperature $T_{c}$ were found which were unexpected for the sample geometry and parameters of the experiment. The plateaus are assumed to be subharmonics of a superconducting gap and arise due to the multiple Andreev reflection and strong quasiparticle overheating in the wire nonequilibrium region. The plateaus are evidence of coexistence of superconductivity and dissipation in such wires. The results presented in the paper could not be described by existing theories.
\end{abstract}

\section{INTRODUCTION}

Switching from the superconducting (S) to normal (N) state and vice-versa and electron transport in a nonequilibrium resistive state in an external current controlled superconducting wire of small cross-section is a topical object for investigation. Such a wire can be used as a single-photon detector [1] or a key element in superconducting quantum interference device (SQUID) [2], phaseslip flux qubit [3, 4], and highly efficient rectifier of ac voltage $[5,6]$.

It is known that $\mathrm{SN}(\mathrm{NS})$ switching in a superconducting quasi-one-dimensional wire (with transverse dimensions smaller than the doubled Ginzburg-Landau temperature-dependent coherence length $2 \xi(T)$ ) currentcarrying occurs due to thermally activated and quantum fluctuations of the superconducting order parameter leading to the appearance of the phase slip centers (PSC) [7, 8]. In the PSC core of the $2 \xi(T)$ size the modulus of the order parameter periodically tends to zero while its time-averaged value is non-zero. A thermally activated phase slip center gives rise to a large number of nonequilibrium quasiparticles which are distributed along the doubled length of quasiparticle diffusion $2 \lambda_{Q}(T)$ (external PSC size) [7, 9]. A wire containing a phase slip center is in the nonequilibrium state in which a superconducting and a dissipative quasiparticle currents coexist.

The presence of quasiparticle current in a long wire with several phase slip centers is supported by virtually linear sections on the curve of current dependence of voltage $V(I)$ with a differential resistance $d V / d I$ multiple to the resistance of one phase slip center. The average direct superconducting current (excess superconducting current $\left.I_{\text {exp }}\right)$ in the wire is determined by the point where prolonged linear sections and the current axis intersect [9].

Constant voltage plateaus (integers with $n=1$ and the Shapiro fractional steps) which appear at $V=m h f / 2 n e$ ( $e$ is the elementary charge, $n$ and $m$ are integers, $h$ is the Planck's constant) on the $V(I)$ curves of superconducting structures with a weak link [10, 11] and quasi- one-dimensional wires [12] under microwave irradiation at the frequency $f$ are evidence of alternating superconducting current in the region of the phase slip center.

A nonequilibrium electron transport in SNS junctions at $V<2 \Delta / e$ ( $\Delta$ is the equilibrium value of the superconducting order parameter (energy gap) in reservoirs) undergoes modification due to the multiple Andreev reflection (MAR) 13 15. In a quasi-one-dimensional superconducting wire, a phase slip center play the role of weak link because the time-averaged modulus of the order parameter (effective superconducting gap) in the core of the phase slip center is smaller in value than the order modulus outside the core of the phase slip center. During MAR, quasiparticles between two superconducting reservoirs are reflected from time to time from the reservoirs obtaining an energy of $n e V>2 \Delta$ which suffices to leave the junction. Multiple crossing of the central junction region by quasiparticles enhances nonequilibrium processes to cause a strong quasiparticle diffusion and strong quasiparticle (or Joule) overheating in the central region of the SNS junction or the phase slip center. The MAR and quasiparticle overheating [13 16] lead to the appearance of current singularities (subharmonics of the superconducting gap) on $V(I)$ curves at $V=2 \Delta / n e$.

MAR and gap subharmonics are described theoretically in the case of ballistic junctions with mean free path for quasiparticles $l$ larger than the junction normalregion length $L$ [13], as well as in the case of diffusive SNS junctions $(l<<L)[14[16]$. The most pronounced singularities can be expected in a short SNS junction [14]. The singularities are poorly visible on the $V(I)$ curves but are fairly distinct on the dependences of $d V / d I$ and $d I / d V$ on $V$. The known theories expect that in [17] do not predict these singularities as voltage plateaus on $V(I)$ curves.

Recently the authors of [17] reported the observation of almost constant-voltage plateau on $V(I)$ curves of short ( $L$ length in the range $\xi(T)<<L<<\lambda_{Q}(T)$ ) quasi-one-dimensional superconducting current-carrying wires placed in magnetic field $B$ at $T$ much lower than $T_{c}$. The plateaus were due to MAR and quasiparticle 
TABLE I: $L_{w}$ - total length of the current wire, $L_{n}$ - length of the central part of the wire (or narrowing), $w_{w}-$ width of the wide part of the wire, $w_{n}$ - width of the central part of the wire (or narrowing), $d$ - film thickness, $R_{N}$ - wire resistance in the $\mathrm{N}$ state, $l$ - mean free path of electrons for wires

\begin{tabular}{lccccccccc}
\hline wire & $L_{w}(\mu \mathrm{m})$ & $L_{n}(\mu \mathrm{m})$ & $w_{w}(\mu \mathrm{m})$ & $w_{n}(\mu \mathrm{m})$ & $d(\mathrm{~nm})$ & $T_{c}(\mathrm{~K})$ & $R_{N}(\Omega)$ & $\xi(0)(\mu \mathrm{m})$ & $l(\mathrm{~nm})$ \\
\hline WA & 70 & 8 & 0.46 & 0.46 & 32 & 1.407 & 17 & 0.14 & 16 \\
WB & 70 & 6.6 & 0.5 & 0.28 & 20 & 1.454 & 69 & 0.1 & 9 \\
WC & 70 & 0.9 & 1.6 & 0.28 & 29 & 1.355 & 4.4 & 0.12 & 13 \\
\hline
\end{tabular}

overheating in the phase slip center formed in the wire. The authors [17] claim "the universal character" of the states with voltage plateaus because the plateau was observed for all samples at $V_{0} \approx(0.43 \pm 0.05) \Delta(0) / e$ (where $\Delta(0)=1.764 k T_{c}$ is the equilibrium superconducting energy gap at $T=0)$. The plateaus are evidence that a superconducting and a dissipative quasiparticle currents coexist in the wire.

SN(NS) switching in short wires are measured in [17. Though, as far as we know there's been no studies of $\mathrm{SN}(\mathrm{NS})$ switching in the case of direct current flowing through a rather long (total length $L_{w} \approx 6-10 \lambda_{Q}(T)$ ) quasi-one-dimensional superconducting wire, while $V$ has been taken from a center short (with length $L_{n}$ satisfying a condition $\left.\xi(T)<L_{n}<\lambda_{Q}(T)\right)$ part of the wire.

In this work we present the measured $V(I)$ curves of three relatively long wires of different geometries (the sketches of the wire central part are given in the insets of Figs. 1, 3, and (4) placed in the field $B$ perpendicular to the substrate surface at $T$ slightly below $T_{c}$. The wires were prepared by thermal deposition of a thin aluminum film onto a silicon substrate by the lift-off technique of electron-beam lithography. Thickness and surface of the wires was checked by an atomic force microscope. The wire parameters are given in the table.

\section{SAMPLES AND EXPERIMENTAL PROCEDURE}

A direct current $I_{d c}$ through $I_{1}$ and $I_{2}$ wires (the insets of Figs. 1, 3, and 4) was applied to $70 \mu \mathrm{m}$ long current wires $w_{w}$ wide which had either no narrowing (wire WA) or a short narrowing $w_{n}$ wide in the wire center (wires $\mathrm{WB}$ and WC). Voltage $V$ was measured in the central part of the wire between the $V_{1}$ and $V_{2}$ wires (the insets of Figs. 1, 3, and (4). The potential wires were $70 \mu \mathrm{m}$ long with the width equal to the width of the wire central part. The large length of current and potential wires allows the decrease of the effect of $15 \mu \mathrm{m}$ wide superconducting leads (which continue the wires) on the electron transport in the wire center.

According to the theories known to these authors, theory in [17] included, gap subharmonics cannot be expected to reveal themselves as voltage plateaus on the $V(I)$ curves for the wires at measurement parameters used in this experiment. However, a number of plateaus of non-universal character were found, which suggests the coexistence of excess superconducting and dissipative quasiparticle currents in a relatively long wire.

The value of $l$ was determined from a refined theoretical expression [18] $\rho l=5.1 \times 10^{-16} \Omega \mathrm{m}^{2}$ where $\rho$ is the wire resistivity. Because $l<<\xi_{0}$ for the wires studied (where $\xi_{0}=1.6 \mu \mathrm{m}$ is the length of superconducting coherence of pure aluminum at $T=0 \mathrm{~K}$ ), the Ginsburg-Landau length at $T$ slightly below $T_{c}$ was calculated by expression $\xi(T)=\xi(0)\left(1-T / T_{c}\right)^{-1 / 2}$ (here $\left.\xi(0)=0.85\left(\xi_{0} l\right)^{1 / 2}\right)$ [9]. In the temperature range of the experiment the wire was quasi-one-dimensional and $\lambda_{Q}(T)=7-12 \mu \mathrm{m}$.

\section{RESULTS AND DISCUSSION}

It was found that the $V(I)$ curves in a general case exhibit a hysteresis depending on the direction of current sweep. The $V(I)$ curve of the WA wire measured at $B=0$ and $T=1.34 \mathrm{~K}$ displays two superconducting critical currents: a switching current $I_{s}$ at which $V$ appears in the wire central part as $I$ increases and a retrapping superconducting current $I_{r}$ at which $V$ disappears with decreasing current $I$ (the upper inset of Fig. 11). It was also found that $I_{s}$ is equal to the Ginsburg-Landau deparing current $I_{G L}$ and exceeds $I_{r}$ which has a smaller value due to a quasiparticle (or Joule) wire overheating. The hysteresis in the $V(I)$ curves disappears in strong fields. In the zero field (the upper inset of Fig. 1) and weak fields the SN switching occurs abruptly as current increases due to strong overheating of the wire. The object of this study was an extended NS switching. Figures 114 (except the upper inset of Fig. 11) shows the branches of $V(I)$ curves corresponding to NS switching.

Figure1 1 presents the $V(I)$ curves for the WA wire measured at $B=0$ and $T$ slightly below $T_{c}=1.407 \mathrm{~K}$. At the beginning of the NS switching, the $V(I)$ curves exhibit a non-monotonic behavior as maxima (curves 1-3). This behavior is caused by the competition of overheating and cooling of the nonequilibrium region in the wire center. The maximum corresponds to an excess quasiparticle overheating. Earlier, $V(I)$ curves with overheating effects were also observed in aluminum microbridges [19].

Moreover, $V(I)$ curves (Fig. 1 and the inset of Fig. 1) display steps of almost constant voltage (voltage 


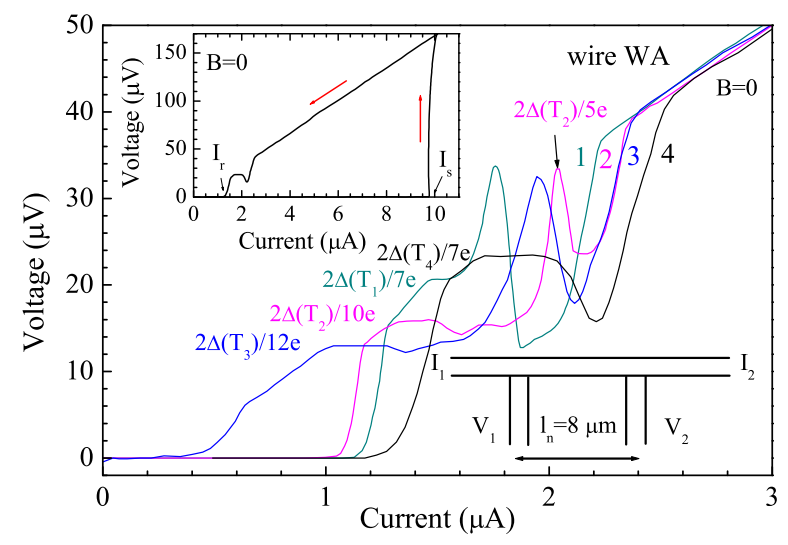

FIG. 1: (Color online) $V(I)$ curves for the WA wire, measured with decreasing current in $B=0$ at $T: T_{1}=1.347$ curve $1, T_{2}=1.344-2, T_{3}=1.341-3, T_{4}=1.340 \mathrm{~K}-4$. The critical temperature is $T_{c}=1.407 \mathrm{~K}$. The corresponding voltages $V_{p l}=2 \Delta(T) / n e(n=7,10,12$, and 7 for curves 1-4, respectively) are given near the plateau. The arrow indicates the maximum at $V=2 \Delta\left(T_{2}\right) / 5 e$. The insert at the top: the $V(I)$ curve, recorded with increasing and decreasing current in $B=0$ at $T=1.34 \mathrm{~K}$; the insert at the bottom: the sketch of the central part of the WA wire (not to scale).

plateaus). The voltage $V_{p l}$ at which plateaus occur depends on $T$. As the current $I$ decreases the plateau usually transforms into a virtually linear section which ends by an abrupt drop to a state close to the superconducting state. We believe that the excess superconducting current and linear sections on the $V(I)$ curves of the studied wires as well as on the $V(I)$ curves of the wires reported in [12] evidently suggest the presence of the phase slip center in the wire central part. The $V(I)$ curves were found independent of the thermocycling: curves 1 and 2 were measured in one cycle, curves 3 and 4 in another cycle (Fig. 1). Figure1 shows that the $V(I)$ curves (curves 3 and 4) measured at virtually equal temperatures reveal certain lack of coincidence when current and potential wires interchange their places (curve 4).

We supposed that the plateaus arise due to MAR and quasiparticle overheating in the phase slip center or a short SNS junction formed in the wire center during $\mathrm{SN}(\mathrm{NS})$ switching. In our case of a long wire, phase slip center and a self-forming short SNS junction differ in that the time-averaged gap in the SNS junction center is smaller than the gap in the core of the phase slip center because of strong overheating of the SNS junction. It was expected that a plateau would appear at voltages $V_{p l}=2 \Delta(T, B) / n e$, where $\Delta(T, B)$ is the almost equilibrium superconducting gap depending on $T$ and $B$ taken in the region outside the PSC core or the SNS junction center. This region in wires with narrowing corresponds to the place where narrow parts of

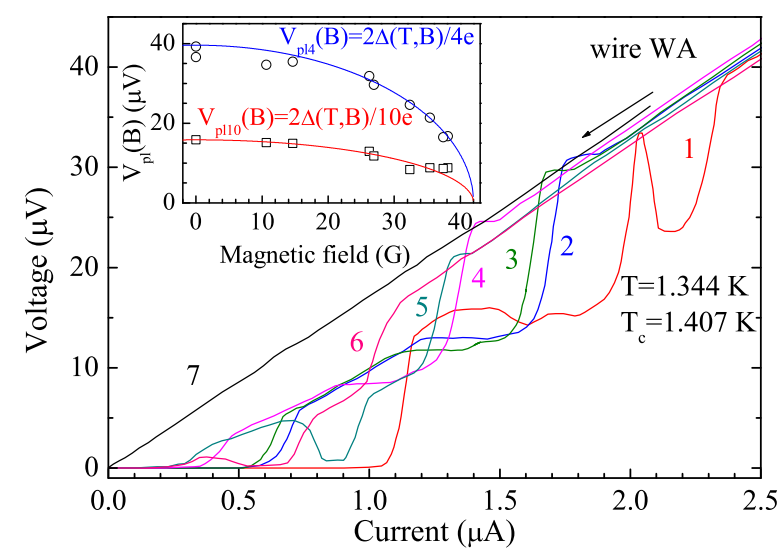

FIG. 2: (Color online) $V(I)$ data for the WA wire, measured at $T=1.344 \mathrm{~K}$ in the magnetic field $B: 0$ - curve $1,26.2-2$, 26.9 - 3, 32.3 - 4, 35.4 - 5, 37.4 - 6, $49.4 \mathrm{G}$ - curve 7 . The critical temperature is $T_{c}=1.407 \mathrm{~K}$. The insert: $V_{p l}(B)$ functions. The experimental data (circles and squares) are approximated by theoretical curves (lines) $2 \Delta(B, T) / n e$ (where $n$ is equal to 4 and 10 for circles and squares, respectively).

current and potential wires intersect. Note that current singularities in SNS junctions are usually determined by $\Delta(T, B)$, taken in the region of wide superconducting leads [13 15]. The gap for the quasi-onedimensional $\mathrm{Al}$ superconducting wire was found from the expression $\Delta(T, B)=\Delta(T)\left(1-\left(B / B_{m t h}(T)\right)^{2}\right)^{1 / 2}$, where $\Delta(T)=1.74 \Delta(0)\left(1-T / T_{c}\right)^{1 / 2}$ is the temperaturedependent gap in the zero field at $T$ slightly below $T_{c}$ and $B_{m t h}(T)$ is the calculated temperature-dependent maximum field at which the gap becomes zero [9].

In Fig. 1 the voltage plateaus, considered to be subharmonics of the gap, are observed at $V_{p l}=2 \Delta(T) / n e$ (where $T$ are temperatures corresponding to curves 1-4, $n$ is defined as an integer close to fitting parameter and equal to $7,10,12$, and 7 for curves $1-4$, respectively). Thus, the plateaus on the curves can correspond to different values of $n$. In addition, splitting of the plateau into two plateaus with close $V_{p l}$ voltages is seen on the curves 2 , and 3. A splitting in subharmonic particularities (not in a form of a plateau) of the gap corresponding to $n=2$, and 4 was found earlier in diffusive SNS structures [16].

Figure 2 presents the $V(I)$ curves for the wire WA measured in different fields at $T=1.344 \mathrm{~K}$. One of the curves exhibits up to two plateaus which are the gap subharmonics. We believe that the plateaus seen in the beginning of the NS switching are supposed to arise due to the appearance of a short self-forming SNS junction in the wire center whereas other plateaus arising at lower currents are caused by phase slip centers. As current $I$ decreases the plateau caused by the phase slip center 


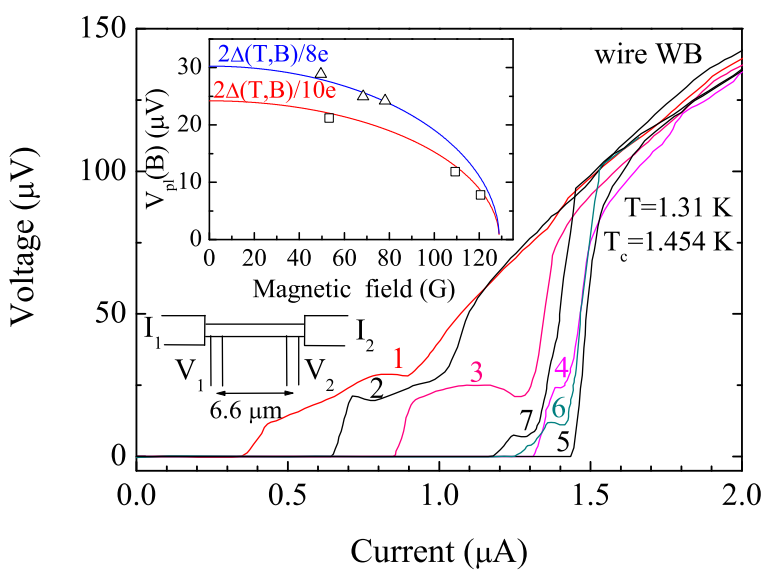

FIG. 3: (Color online) $V(I)$ curves for the WB wire, recorded at $T=1.31 \mathrm{~K}$ in the fields $B: 49.6$ - curve $1,53.2-2,68.4-3$, 78.1 - 4, 82.6 - 5, 109.3 - 6, $120.5 \mathrm{G}$ - curve 7 . The critical temperature is $T_{c}=1.454 \mathrm{~K}$. The plateaus correspond to the voltages $2 \Delta(B, T) / 8 e$ (for curves 1,3 , and 4 ) and $2 \Delta(B, T) / 10 e$ (for curves 2,6 , and 7 ). The left insert: Triangles and squares - measured $V_{p l}(B)$ functions. Lines - the theoretical curves $2 \Delta(B, T) / n e$ with $n=8$ and 10 approximate the measured data. The insert at the bottom: the sketch of the WB wire (not to scale).

becomes almost linear segment. As the field increases, the peak on the $V(I)$ curves (curve 1) related to the beginning of the NS switching disappears because the field reduces the gap in the region outside the wire center to cause a more efficient cooling of overheated quasiparticles [20]. The character of the curves again becomes non-monotonic in high fields in the region close to the $\mathrm{S}$ state (curves 5 and 6 ). The maxima on curves 5 and 6 are related to the reappearance of the phase slip center with a current decrease, which had earlier disappeared in higher fields. At $B=37.4 \mathrm{G}$ (curve 6 ) close to the maximum field $B_{m}(T=1.344 K)=42 \mathrm{G}$, both plateaus disappear because of a strong decreasing of the gap. At $37.4 \mathrm{G}$ the gap is not equal to zero yet therefore the phase slip center remains in the wire center (as is evidenced by the linear section of curve 6 and an excess superconducting current in the region close to the $\mathrm{S}$ state (curve 6)). Thus, the formation of a phase slip center or a SNS junction in the wire center at certain current (voltage) values is an essential condition for plateau observation.

The inset of Fig. 2 suggests that the plateau (Fig. 2) at voltages $V_{p l}(B)$ depending on field can be regarded as the gap subharmonics with $n=4$ and $n=10$. It is seen that the plotted $V_{p l}(B)$ functions (circles and squares) taken from the experimental $V(I)$ curves $(T=1.344 \mathrm{~K})$ of the WA wire can be fitted by the theoretical curves $V_{p l 4}(B)=2 \Delta(T, B) / 4 e$ and $V_{p l 10}(B)=2 \Delta(T, B) / 10 e$, where $\Delta(T, B)=\Delta(T)\left(1-\left(B / B_{m}\right)^{2}\right)^{1 / 2}$ and $B_{m}=42 \mathrm{G}$

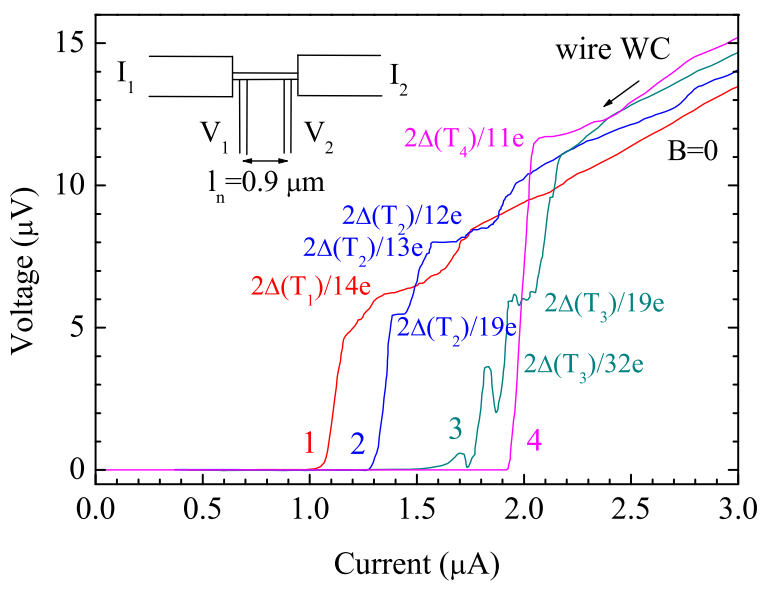

FIG. 4: (Color online) $V(I)$ curves for the WC wire in $B=0$ at $T: T_{1}=1.334$ - curve $1, T_{2}=1.327-2, T_{3}=1.320-3$, $T_{4}=1.312 \mathrm{~K}-4$. The critical temperature is $T_{c}=1.355 \mathrm{~K}$. The corresponding voltages $V_{p l}(T)=2 \Delta(T) / n$ are given near the plateau. The insert: the $\mathrm{WC}$ wire sketch.

is the only fitting parameter for the maximum magnetic field close to the calculated value of $B_{m t h}(T=$ $1.344 K)=38 \mathrm{G}$.

Figure 3 shows the $V(I)$ curves for the WB wire (with narrowing) measured in different fields at $T=1.31 \mathrm{~K}$. As the field increases the retrapping superconducting current $I_{r}$ increases (curves 1-5, a similar anomalous behavior of $I_{r}$ was found in the "magnetic-field-induced superconductivity" and "anti-proximity effects" 21, 22]); at further increasing of the field $I_{r}$ demonstrates a usual decreasing (curves 6 , and 7). One of the curves exhibits a short horizontal segment of almost constant voltage or one slightly tilted segment. We regard both the segments as plateaus. One curve exhibits one plateau. The plateaus depend on field and are observed (curves 1-4, 67) up to the fields close to the calculated maximum magnetic field in the wire narrowing $B_{m n t h}(T=1.31 \mathrm{~K})=$ $129 \mathrm{G}$. The plateau is not observed on curve 5 recorded in the field close to the maximum field in the wide part of the wire $B_{m w t h}=73 \mathrm{G}$.

The inset of Fig. 3 shows that plateaus (Fig. 3) at $V_{p l}(B)$ can be treated as the gap subharmonics with $n=8$, and $n=10$. It is seen that the plotted $V_{p l}(B)$ (triangles and squares) data derived from the measured $V(I)$ curves $(T=1.31 \mathrm{~K})$ for the WB wire fit the theoretical curves $V_{p l 8}(B)=2 \Delta(T, B) / 8 e$ and $V_{p l 10}(B)=2 \Delta(T, B) / 10 e$, where $\Delta(T, B)=\Delta(T)(1-$ $\left.\left(B / B_{\text {mnth }}(T)\right)^{2}\right)^{1 / 2}$ at $T=1.31 \mathrm{~K}$.

Figure 4 exhibits the $V(I)$ curves for the WC wire recorded in the zero field at temperatures $T$ slightly below $T_{c}=1.355 \mathrm{~K}$. Short almost horizontal segments along with slightly tilted segments of approximately constant 
voltage (plateaus) are seen on the $V(I)$ curves. One curve can display not less than three plateaus (curve 2). We found the $V_{p l}$ is a function of the temperature. We believe that the plateaus (Fig. 44) can be treated as the gap subharmonics $2 \Delta(T) / n$ with $n=11,12,13,14,19$, and 32. The specific features of the WC wire are a short narrowing of the $L_{n}<2 \xi(T)$ length in the studied range and a large width $(1.6 \mu \mathrm{m})$ of the major wide part of the wire. The wire was quasi-one-dimensional at $T>1.32 \mathrm{~K}$. At this geometry, a nonequilibrium region in the narrowing is more appropriate to regard as Josephson (a short SNS) junction rather than a phase slip center [11].

The formation of a SNS junction in the wire center instead of a phase slip center is supported by the fact that plateaus disappeared at mutual exchange of current and potential wires. Apart from plateaus, at high voltages $V$, when a state of the wire narrowing is approaches to the a normal state and the rest of the wire remains superconducting, we can observe a number of subharmonic singularities (not in a form of a plateau) on $V(I)$ curves (the right part of Fig. (4) and $d I / d V(V)$ curves (not shown here). For instance $d I / d V$ as a function of $V$ with the parameters of the $V(I)$ curve (curve 4 in Fig. 4) shows a successive array of the singularities corresponding to $n=3-11$, where the singularity with $n=11$ is attributed to the plateau on the $V(I)$ curve. Singularities with $n>11$ are invisible because they relate to the vertical segment of the $V(I)$ curve.

A question could be asked: why could we not see a full successive array of subharmonics (starting with $n=1$ ) and why are subharmonics that are revealed as plateaus so scarce or why could we see a plateau corresponding to a large subharmonic number $(n=32)$.

We answer as follows. Because the $V(I)$ curves of our wires were recorded in a bias direct current mode, that is why instead of subharmonic singularities that are observed at low $V$ in the region of negative values for $d V / d I$ in a bias voltage mode, we see almost vertical segments of the $V(I)$ curves. At higher $V$ values in the resistive region close to the normal state, a successive subharmonic array might also not be seen as SNS junction appearance is improbable due to an overheating of the wire center and of the adjacent parts of the wire close to the center. That is true with the exception of the $\mathrm{WC}$ wire center (Fig. 4) with wide adjacent parts that remain superconducting when measured in the zero field.

We found experimentally that plateaus are registered in a restricted voltage interval relating to a nonequilibrium part on $V(I)$ curves, when a steady SNS junction or a phase slip center is appeared in the middle of the wire, as is verified by a linear segment of the $V(I)$ curve. Only subharmonics with given numbers $n$ (visible as plateaus at voltages $\left.V_{p l}=2 \Delta(T, B) / n e\right)$ get into this interval. The upper boundary of this interval $V<2 \Delta(T, B) / e$ is defined by multiplication of the wire resistance in normal state by the value of the current at which the wire resistance begins sharply drop with decreasing current. A considerable decrease of the $V$ due to wire overheating leads to a decrease in maximum possible value of $V_{p l}(B)$ and consequently to the increase of a maximum number $n$ of the visible subharmonics.

To observe multiple Andreev reflection with large numbers $n$ in diffusive SNS contacts, it is necessary that $l_{\text {in }}$ [14] inelastic scattering length (in our case, $l_{\text {in }}$ corresponds to twice the length of quasiparticle diffusion $\left.2 \lambda_{Q}(T) \approx 14-24 \mu \mathrm{m}\right)$ is much longer than the length of the normal region. This requirement, written as $l_{\text {in }}=2 \lambda_{Q}(T)>>2 \xi(T)$, is fulfilled here.

We believe subharmonic features, including plateaus, arise due to MAR and quasiparticle overheating in a short SNS contact or in a phase slip center. To observe the plateau, a stronger overheating is necessary, which can be realized in aluminum and zinc wires due to the long electron-phonon relaxation times. So these relaxation times for aluminum and zinc equal to $\tau_{E}=1.3 \times 10^{-8} \mathrm{~s}$ and $\tau_{E}=9.3 \times 10^{-8} \mathrm{~s}$, respectively, and exceed by a factor of two the relaxation time for tin $\tau_{E}=9.3 \times 10^{-11} \mathrm{~s}$ 23].

The coupling of the alternating Josephson current with the external resonator, randomly formed in the measuring electrical circuit, or the internal resonator in the wire, cannot cause a plateau in the observed voltage range of 5-40 $\mu \mathrm{V}$ (Figs. 1 14). Since the interval of Josephson frequencies corresponding to this voltage range does not intersect with the expected frequency intervals of the external and internal resonators. In addition, it could be expected that the voltage, at which a plateau occurs, caused by resonators will not clearly depend on $T$ and $B$, whereas in our case the voltage at which a certain plateau is observed depends on $T$ and $B$.

Below the comparison is given of the results obtained in this work with those reported in [17].

1. In the zero field, our $V(I)$ curves had a hysteresis depending on the direction of the current sweep, while the switching current $I_{s}$ was equal to $I_{G L}$ and the retrapping current $I_{r}$ could have values 8-15 times smaller than $I_{G L}$. Whereas in [17] $V(I)$ curves were not hysteresis, while $I_{s}$ and the retrapping current $I_{r}$ coincided and were 30-50 times smaller than $I_{G L}$. The very low $I_{s} / I_{G L}$ value means that the electron transport in wires 17] is strongly influenced by internal fluctuations and external noises, leading to premature wire switching with increasing external current from the $\mathrm{S}$ state to the $\mathrm{N}$ state and the absence of a $V(I)$ curve hysteresis. The very low value of $I_{r} / I_{G L}$ indicates a strong overheating of the current-carrying wire [17], leading to a later (at low currents) wire switching with a decrease in the current from the $\mathrm{N}$ state to the $\mathrm{S}$ state. So, fluctuations, external noises and overheating are stronger in wires [17] than in our wires.

2. The voltage plateau on the $V(I)$ curves of our wires was observed at $T$ slightly below $T_{c}$. The curves of [17] 
exhibit the plateau at $T$ much lower than $T_{c}$.

3. The plateaus were stable with time. In [17] the plateaus were reported bistable. The bistability, in our opinion, arises because of strong internal fluctuations, external noises and overheating on the wires of [17].

4. We could observe as many as two plateaus on one $V(I)$ curve (Figs. 2, and 4) caused by MAR both in the SNS junction and phase slip center. The experiment and theory in [17] display only one plateau due to MAR in the phase slip center.

5 . One of the important differences between the results of this work and those in [17] is the following. The states with plateau observed in this work are not universal because the voltage $V_{p l}(T, B)=2 \Delta(T, B) / n e$ at which the plateau arises depends on $T, B$, and the gap subharmonic gap number $n$. Plateaus were observed which correspond to large $n$ (up to $n=32$ ). The authors of [17] state that states with plateau are of universal character. The theory proposed in 17] predicts that the plateau can be observed at voltages $V_{0}^{t h}=2 \Delta_{e f f} / e \approx 0.34 \Delta(0) / e$ independent of $T, B$, and $n$, where $\Delta_{\text {eff }}$ is an average nonequilibrium suppressed gap in the wire center. The excess of the experimental ratio $V_{0} /(\Delta(0) / e)$ over the theoretical ratio $V_{0}^{t h} /(\Delta(0) / e)=0.34$ reaches $35 \%$ for the zinc wire (Sample A) and $44 \%$ for the aluminum wire (Sample D) 17]. Thus, there is a difference between the theory [17] and the measurements [17].

We propose an alternative calculation of the voltage at which there is a plateau in [17], using the expression $V_{p l}(T, B)=2 \Delta(T, B) / n e$ for non-universal plateaus from our work. In the general case, the value of the temperature-dependent gap in the zero field should be obtained from the Bardeen-Cooper-Schrieffer theory and not from the expression $\Delta(T)$, valid near $T_{c}[9]$. It is easy to check that for the wires of the Sample A and Sample D [17], the values of $V_{p l}(T, B)$ calculated within our model at $T=0.45 \mathrm{~K}$ coincide with the measured values 17 with an accuracy of $1 \%$ if $n=4$. Thus, the plateaus [17] can be understood as the gap subharmonics with $n=4$.

Since the plateau [17] disappears in weak fields $B>$ $20 \mathrm{G}$ and near Tc, the "universality" of the plateau (i.e., the independence of the voltage value at which the plateau is observed from $T$ and $B$ ) cannot be experimentally verified for a wide range of temperatures and fields. This "universality" has been experimentally proven [17] with an accuracy of $\approx 12 \%$ for a narrow field interval $B<20 \mathrm{G}$ (where the expected change in the gap in our model is $\approx 1 \%$ ) and the temperature interval $0<T<0.64 T_{c}$ (where the expected change in the gap in our model is $\approx 12 \%$ ).

6. The field suppression of the gap in wide wires far from the wire center did not affect the plateau. The plateau was observed in a wide range of fields. The plateau on the $V(I)$ curves of the WB wire (with narrowing) was observed when the gap in the main wide part of the wire was set to zero and disappeared when the gap in the wire narrow part was zero. In the WA wire (without narrowing) the plateau disappeared when the gap was set to zero in the region outside the SNS junction or in the PSC core. In [17] the range of fields at which the plateau exists was very narrow because of a strong effect of wide superconducting leads. The plateau disappeared [17] when the gap in the superconducting lead slightly decreased due to a weak field or when $T$ approached $T_{c}$.

7. The $V(I)$ curves of the WA wire (without narrowing) displayed the plateau in the absence of effects similar to those described in 21, 22]. Moreover, the $V(I)$ curves of the WB wire (with narrowing) exhibited the plateau both in the presence of the effects described in 21, 22. (curves 1-4, Fig. 3) and without such effects (curves 5-7, Fig. 3). This disagrees with the statement 17] that the appearance of the state with a voltage plateau is strongly connected with these effects [21, 22].

\section{CONCLUSION}

To sum up, we observed a new effect which proves the coexistence of superconductivity and dissipation in the nonequilibrium state of relatively long quasi-onedimensional current-currying aluminum wires. The effect observed radically differs from the effect reported in [17]. In this work non-universal plateaus of almost constant voltage on the $V(I)$ curves for the wires used which were recorded at a decreasing current in magnetic field at $T$ slightly below $T_{c}$ were found. These plateaus were quite unexpected for these wires at the external parameters of the experiment. One of the curves could exhibit as many as four plateaus. Commonly, subharmonic features of the superconducting gap caused by multiple Andreev refelection and quasiparticle overheating in the nonequilibrium region display themselves as current singularities on the $V(I)$ curves.

We found that these subharmonic features of the gap can appear as plateaus at voltages $V_{p l}(T, B)=$ $2 \Delta(T, B) / n e$, with $n$ having certain integer values depending on $T, B$, and $V$. We believe that a very strong quasiparticle overheating in the PSC core or SNS junction, caused by a long time of electron-phonon relaxation in aluminum wires, is necessary for the plateau to appear. The observation of the plateau depends on the wire geometry. For example, the plateau exists up to strong fields close to the maximum fields $H_{m} \propto w_{w}^{-1}$ for wires of constant width $w_{w}$ and $H_{m n} \propto w_{n}^{-1}$ for wires with narrowing $w_{n}$, respectively. Splitting of the plateau (Fig. 1) and non-monotone character of $V(I)$ curves were observed upon current variation caused switching of the wire between different states as a result of competition between quasiparticle overheating and cooling in the wire center. In the region close to the $\mathrm{N}$ state subharmonics are seen in Fig. 4 as weak current singularities (not 
plateaus).

\section{ACKNOWLEDGMENTS}

The work was supported by the Presidium of Russian Academy of Sciences (research program "Challenging Problems of Low Temperature Physics"). We thank V. Tulin, D. Vodolazov, A. Melnikov, M. Skvortsov for fruitful discussions and O. Trofimov for technical assistance.

* Electronic address: kvi@iptm.ru

[1] G. N. Gol'tsman, O. Okunev, G. Chulkova, A. Lipatov, A. Semenov, K. Smirnov, B. Voronov, A. Dzardanov, C. Williams, and R. Sobolewski, Appl. Phys. Lett. 79, 705 (2001).

[2] A. Barone and G. Paterno. Physics and Application of the Josephson Effect. John Willey and Sons, New York, 1982.

[3] J. E. Mooij and C. J. P. M. Harmans, New J. Phys. 7, 219 (2005).

[4] O. V. Astafiev, L. B. Ioffe, S. Kafanov, Yu. A. Pashkin, K. Yu. Arutyunov, D. Shahar, O. Cohen, and J. S. Tsai, Nature (London) 484, 355 (2012).

[5] S. V. Dubonos, V. I. Kuznetsov, I. N. Zhilyaev, A. V. Nikulov, A. A. Firsov, JETP Lett. 77, 371 (2003) (original Russian text: S. V. Dubonos, V.I. Kuznetsov, I. N. Zhilyaev, A. V. Nikulov, A. A. Firsov, Pis'ma v Zhurnal Eksperimental'noi i Teoreticheskoi Fiziki 77, 439 (2003)), https://doi.org/10.1134/1.1581963, arXiv:cond-mat/0303538.

[6] V.I. Kuznetsov, A.A. Firsov, S. V. Dubonos, $\begin{array}{lllll}\text { Phys. Rev. B } & \mathbf{7 7}, & 094521 & \text { (2008), }\end{array}$ https://doi.org/10.1103/PhysRevB.77.094521.
[7] B. I. Ivlev and N. B. Kopnin, Sov. Phys. Usp. 27, 206 (1984).

[8] A. D. Zaikin, D. S. Golubev, A. van Otterlo, and G. T. Zimanyi, Phys. Rev. Lett. 78, 1552 (1997).

[9] V.V. Schmidt, The Physics of Superconductors (Eds. P. Muller and A.V. Ustinov, Springer-Verlag, BerlinHeidelberg, 1997).

[10] S. Shapiro, Phys. Rev. Lett. 11, 80 (1963).

[11] K. K. Likharev, Rev. Mod. Phys. 51, 101 (1979).

[12] V. I. Kuznetsov and V.A. Tulin, J. Exp. Theor. Phys. 86, 745 (1998) (original Russian text: V.I. Kuznetsov and V.A. Tulin, Pis'ma v Zhurnal Eksperimental'noi i Teoreticheskoi Fiziki 113, $1364 \quad$ (1998)), https://doi.org/10.1134/1.558535, arXiv:cond-mat/0407464.

[13] M. Octavio, M. Tinkham, G. E. Blonder, and T. M. Klapwijk, Phys. Rev. B 27, 6739 (1983).

[14] A. Bardas and D. V. Averin, Phys. Rev. B 56, R8518 (1997).

[15] E.V. Bezuglyi, E.N. Bratus', V.S. Shumeiko, G. Wendin, and H. Takayanagi, Phys. Rev. B 62, 14439 (2000).

[16] R. Taboryski, J. Kutchinsky, J. B. Hansen, M. Wildt, C. B. Sorensen, and P. E. Lindelof, Superlattices and Microstructures 25, 829 (1999).

[17] Y. Chen, Y.-H. Lin, S. Snyder, A. Goldman, and A. Kamenev, Nature Physics 10, 567 (2014).

[18] M. Gershenson, and W. L. McLean, J. Low Temp. Phys. 47, 123 (1982).

[19] T. M. Klapwijk, M. Sepers, and J. E. Mooij, J. Low Temp. Phys. 27, 801 (1977).

[20] D. Y. Vodolazov and F. M. Peeters, Phys. Rev. B 85, 024508 (2012).

[21] Y. Chen, S. D. Snyder, and A. M. Goldman, Phys Rev. Lett. 103, 127002 (2009).

[22] M. Tian, N. Kumar, S. Xu, J. Wang, J. S. Kurtz, and M. H. W. Chan, Phys. Rev. Lett. 95, 076802 (2005).

[23] M. Stuivinga, J. E. Mooij, and T. M. Klapwijk, J. Low Temp. Phys. 46, 555 (1982). 